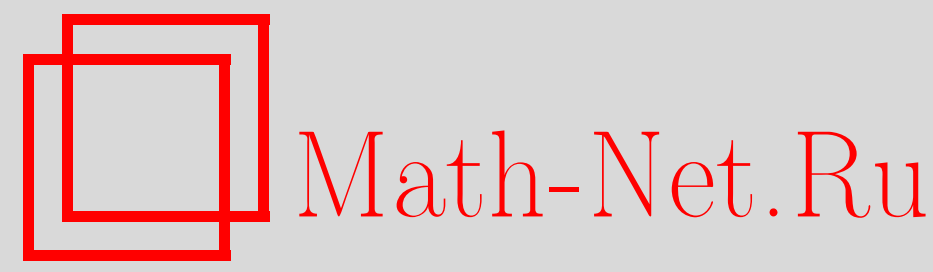

В. И. Коляда, Вложения дробных пространств Соболева и оценки преобразований Фурье, Матем. сб., 2001, том 192, номер 7, 51-72

DOI: https://doi.org/10.4213/sm579

Использование Общероссийского математического портала Math-Net.Ru подразумевает, что вы прочитали и согласны с пользовательским соглашением http://www . mathnet.ru/rus/agreement

Параметры загрузки:

IP : 54.162 .85 .209

26 апреля 2023 г., 05:17:12 
УДК 517.5

\author{
В.И. Коляда
}

\title{
Вложения дробных пространств Соболева и оценки преобразований Фурье
}

\begin{abstract}
В статье изучаются дробные анизотропные пространства Соболева-Лиувилля $L_{p}^{r_{1}, \ldots, r_{n}}\left(\mathbb{R}^{n}\right)$, где $1 \leqslant p<\infty$ и $r_{k}$ - положительные числа. Для функций из этих пространств устанавливаются оценки норм в модифицированных пространствах типа Лоренца и Бесова, определяемых в терминах повторных перестановок. С помощью этих оценок доказьваются неравенства для преобразований Фурье функций из пространства $L_{1}^{r_{1}}, \ldots, r_{n}$.

Статья является продолжением работ автора, в которых аналогичные вопросы изучались в случае целых $r_{k}$.

Применяемые в работе методы основаны на оценках повторных перестановок. Этот подход позволяет упростить доказательства и в то же время получить более сильные резултаты. В частности, существенно упрощается исследование предельного случая $p=1$.

Библиографоия: 19 названий.
\end{abstract}

\section{§1. Введение}

В статье устанавливаются теоремы вложения и оценки преобразований Фурье для функций из дробных анизотропных пространств Соболева-Лиувилля.

Приведем основные определения (подробные пояснения и соответствуюшие ссылки можно найти в [1]).

Ядро Бесселя порядка $\alpha>0$ в $\mathbb{R}^{n}$ определяется как функция, преобразование Фурье которой равно

$$
\widehat{G}_{\alpha}(\xi)=\left(1+4 \pi^{2}|\xi|^{2}\right)^{-\alpha / 2}, \quad \xi \in \mathbb{R}^{n} .
$$

Пусть $1 \leqslant p<\infty$ и $r_{1}, \ldots, r_{n}$ - положительные числа. Через $L_{p}^{r_{1}, \ldots, r_{n}}\left(\mathbb{R}^{n}\right)$ будем обозначать пространство всех функций $f \in L^{p}\left(\mathbb{R}^{n}\right)$, обладаюших следующими свойствами:

(1) если $r_{k} \in \mathbb{N}$, то существует обобщенная частная производная порядка $r_{k}$ по $k$-й переменной $D_{k}^{r_{k}} f \in L^{p}\left(\mathbb{R}^{n}\right)$;

(2) если $r_{k} \notin \mathbb{N}$, то сушествует функция $f_{k} \in L^{p}\left(\mathbb{R}^{n}\right)$ такая, что для почти всех $x \in \mathbb{R}^{n}$

$$
f(x)=\int_{\mathbb{R}} G_{r_{k}}\left(x_{k}-y\right) f_{k}\left(y, \widehat{x}_{k}\right) d y .
$$

Работа выполнена при финансовой поддержке Министерства образования Испании (Sabbatical grant SAB95-0654).

(C) В. И. КолядА 2001 
Заметим, что в случае (2) функция $f_{k}$ определяется однозначно с точностью до значений на множестве $n$-мерной меры нуль и вьполняется неравенство

$$
\|f\|_{p} \leqslant\left\|f_{k}\right\|_{p} .
$$

Будем называть $f_{k}$ бесселевой производной порядка $r_{k}$ функции $f$ по переменной $x_{k}$ и обозначать через $D_{k}^{r_{k}} f$ (подчеркнем, однако, что в случае целого $r_{k}$ это обозначение сохраняется для обычной производной).

Норма в пространстве $L_{p}^{r_{1}}, \ldots, r_{n}\left(\mathbb{R}^{n}\right)$ определяется равенством

$$
\|f\|_{L_{p}^{r_{1}, \ldots, r_{n}}}=\|f\|_{p}+\sum_{k=1}^{\infty}\left\|D_{k}^{r_{k}}\right\|_{p}
$$

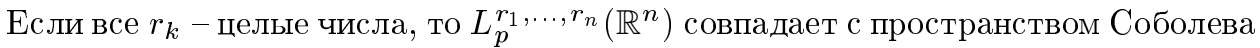
$W_{p}^{r_{1}, \ldots, r_{n}}\left(\mathbb{R}^{n}\right)$. Будем называть $L_{p}^{r_{1}, \ldots, r_{n}}\left(\mathbb{R}^{n}\right)$ дробным пространством Соболева или пространством Соболева-Лиувилля. Заметим, что данное определение отличается от приведенного в монографии [2] лишь в случае, когда $p=1$ и хотя бы одно из $r_{k}$ является целым нечетным числом.

Как обычно, через $C_{0}^{\infty}\left(\mathbb{R}^{n}\right)$ обозначаем множество всех бесконечно дифференцируемых функций с компактным носителем в $\mathbb{R}^{n}$.

Множество $C_{0}^{\infty}\left(\mathbb{R}^{n}\right)$ является всюду плотным подмножсеством в пространстве $L_{p}^{r_{1}, \ldots, r_{n}}\left(\mathbb{R}^{n}\right)$ для любых $1 \leqslant p<\infty, r_{k}>0$.

Для доказательства этого утверждения достаточно использовать $\varepsilon$-усреднения и умножение на “срезающие” функции (см. [3; гл. 5], [4; $§ 27.3],[1])$.

Напомним определение пространств Бесова. Если функция $f$ задана на $\mathbb{R}^{n}$, $1 \leqslant k \leqslant n$ и $r \in \mathbb{N}$, то полагаем

$$
\Delta_{k}^{r}(h) f(x)=\sum_{j=0}^{r}(-1)^{r-j}\left(\begin{array}{l}
r \\
j
\end{array}\right) f\left(x+j h e_{k}\right)
$$

(где $x \in \mathbb{R}^{n}, h \in \mathbb{R}$ и $e_{k}$ - единичный координатный вектор). Предположим, что $f \in L^{p}\left(\mathbb{R}^{n}\right)(1 \leqslant p<\infty)$. Тогда функция

$$
\omega_{k}^{r}(f ; \delta)_{p}=\sup _{0 \leqslant h \leqslant \delta}\left\|\Delta_{k}^{r}(h) f\right\|_{p} \quad(\delta \geqslant 0)
$$

называется частным модулем непрерывности функции $f$ порядка $r$ в $L^{p}$ по переменной $x_{k}$. В приведенных обозначениях при $r=1$ верхний индекс обычно не пишут.

Пусть $1 \leqslant p, \theta<\infty$ и $\alpha_{1}, \ldots, \alpha_{n}$ - положительные числа. Пространством Бесова $B_{p, \theta}^{\alpha_{1}, \ldots, \alpha_{n}}\left(\mathbb{R}^{n}\right)$ называется класс всех функций $f \in L^{p}\left(\mathbb{R}^{n}\right)$, для которых

$$
\|f\|_{B_{p, \theta}^{\alpha_{1}, \ldots, \alpha_{n}}} \equiv\|f\|_{p}+\sum_{k=1}^{n}\left(\int_{0}^{\infty}\left(t^{-\alpha_{k}}\left\|\Delta_{k}^{r_{k}}(t) f\right\|_{p}\right)^{\theta} \frac{d t}{t}\right)^{1 / \theta}<\infty
$$

где $r_{k} \in \mathbb{N}$ и $r_{k}>\alpha_{k}(k=1, \ldots, n)$. При любом выборе целых $r_{k}$ получаются эквивалентные нормы; кроме того, в определении можно заменить нормы конечных разностей соответствуюшими частными модулями непрерывности [2; гл. 4], [5; гл. 4]. 
Настоящая статья является продолжением работ автора [1], [6], [7]. В частности, мы распространяем результаты работ [6], [7] на произвольные значения $r_{k}$ и доказываем некоторые теоремы, анонсированные в обзорной статье [1]. Детальное описание предшествовавших исследований содержится в [1], и мы не будем его воспроизводить.

В работах [1], [6], [7] использовались методы, основанные на оценках невозрастающих перестановок и геометрических неравенствах изопериметрического типа. Отметим, что применение этих методов в теории вложения классов функций восходит к работам П.Л. Ульянова конца 60-х годов (см. [8], [9]). В основе нашей статьи также лежат оценки перестановок, однако здесь мы применяем так называемые повторные перестановки. Функция $f$, заданная на $\mathbb{R}^{n}$, переставляется в невозрастающем порядке сначала по одной переменной, затем по другой и т. д. В итоге получается функция на $\mathbb{R}_{+}^{n}$, невозрастающая по каждой из переменных и равноизмеримая с $|f|$. По-видимому, впервые в теоремах вложения повторные перестановки применялись П. Освальдом [10].

Использование повторных перестановок имеет ряд преимушеств. В частности, оно позволяет сушественно упростить некоторые доказательства и в то же время получить более сильные результаты.

Строгое определение повторной перестановки приведено в $\S 2$. Там же получены основные оценки перестановок, используемые в дальнейшем. Далее, в терминах повторных перестановок определяется пространство типа Лоренца $L_{\mathscr{R}}^{q, p}\left(\mathbb{R}^{n}\right)$. При $q>p$ норма этого пространства $\|\cdot\|_{q, p ; \mathscr{R}}$ сильнее нормы обычного пространства Лоренца $L^{q, p}\left(\mathbb{R}^{n}\right)$ :

$$
\|f\|_{q, p} \leqslant c\|f\|_{q, p ; \mathscr{R}} .
$$

Основные результаты работы содержатся в $\S \S 3-5$. Формулируя эти результаты, будем предполагать, что $r_{1}, \ldots, r_{n}$ - заданные положительные числа и

$$
r=n\left(\sum_{k=1}^{n} \frac{1}{r_{k}}\right)^{-1} .
$$

В $\S 3$ исследуется вложение пространств $L_{p}^{r_{1}, \ldots, r_{n}}$ в пространства Лоренца. Здесь доказывается следующая теорема: если $n \geqslant 2,1 \leqslant p<n / r$ и $q^{*}=n p /(n-r p)$, то для каждой функции $f \in L_{p}^{r_{1}, \ldots, r_{n}}\left(\mathbb{R}^{n}\right)$

$$
\|f\|_{q^{*}, p ; \mathscr{R}} \leqslant c \sum_{k=1}^{\infty}\left\|D_{k}^{r_{k}}\right\|_{p} .
$$

В силу (1.1) отсюда следует вложение

$$
L_{p}^{r_{1}, \ldots, r_{n}}\left(\mathbb{R}^{n}\right) \hookrightarrow L^{q^{*}, p}\left(\mathbb{R}^{n}\right)
$$

Другими методами это вложение было доказано в [1] (для целых $r_{k}-$ в [6]). В свою очередь, из (1.3) выводим, что

$$
L_{p}^{r_{1}, \ldots, r_{n}}\left(\mathbb{R}^{n}\right) \hookrightarrow L^{q^{*}}\left(\mathbb{R}^{n}\right)
$$


Для целых $r_{k}$ - это теорема вложения Соболева; в случае произвольных $r_{k}>0$ и $p>1$ вложение (1.4) доказал П.И. Лизоркин (соответствующие ссылки содержатся в [1]). Для $p=n=1$ (1.4) не имеет места.

В $\S 4$ устанавливается основной результат работы. Здесь мы ограничимся формулировкой его следствия - теоремы о вложении в пространство Бесова.

Предположим, что

$$
1 \leqslant p<q<\infty \text { и } \varkappa \equiv 1-\frac{n}{r}\left(\frac{1}{p}-\frac{1}{q}\right)>0 .
$$

Если $1<p<\infty$ и $n \geqslant 1$, или $p=1$ и $n \geqslant 2$, то

$$
L_{p}^{r_{1}, \ldots, r_{n}}\left(\mathbb{R}^{n}\right) \hookrightarrow B_{q, p}^{\alpha_{1}, \ldots, \alpha_{n}}\left(\mathbb{R}^{n}\right), \quad \alpha_{k}=\varkappa r_{k} .
$$

В случае целых $r_{k}$ это вложение было известно - для $p>1$ оно было доказано В.П. Ильиным [5; теорема 18.12], а для $p=1$ - автором [6].

Заметим, что исследование пространств $L_{p}^{r_{1}, \ldots, r_{n}}$ в случае $p=1$ сопряжено с дополнительньми трудностями (эта проблема подробно обсуждается в [1]). В частности, и для целых $r_{k}$ доказательство вложения (1.5) в [6] при $p=1$ значительно сложнее, нежели в случае $p>1$. Как и в [1], [6], одной из главных задач настояшей статьи является разработка методов, позволяющих охватить все значения $p \geqslant 1$. Подход к этой задаче удается значительно упростить благодаря применению повторных перестановок и связанных с ними специальных операторов (см. (2.13)).

Вложение (1.5) (при $p=1$ ) применяется в $\S 5$ для получения оценок преобразований Фурье функций из пространства $L_{1}^{r_{1}, \ldots, r_{n}}\left(\mathbb{R}^{n}\right)$. В частности, мы распространяем соответствующие результаты из работ [7], [11], [12] на произвольные значения $r_{k}>0$. Приведем одно из следствий: для любой функции $f \in L_{1}^{r_{1}, \ldots, r_{n}}\left(\mathbb{R}^{n}\right)$ $(n \geqslant 2)$

$$
\int_{\mathbb{R}^{n}}|\widehat{f}(\xi)|\left(\sum_{j=1}^{n}\left|\xi_{j}\right|^{r_{j}}\right)^{1-n / r} d \xi \leqslant c \sum_{j=1}^{n}\left\|D_{j}^{r_{j}}\right\|_{1} .
$$

\section{§ 2. Невозрастающие перестановки}

Через $S_{0}\left(\mathbb{R}^{n}\right)$ будем обозначать класс всех измеримых почти везде конечных функций $f$ на $\mathbb{R}^{n}$ таких, что для любого $y>0$

$$
\lambda_{f}(y) \equiv\left|\left\{x \in \mathbb{R}^{n}:|f(x)|>y\right\}\right|<\infty .
$$

Невозрастающей перестановкой функции $f \in S_{0}\left(\mathbb{R}^{n}\right)$ называется невозрастаюшая на $\mathbb{R}_{+} \equiv(0,+\infty)$ функция $f^{*}$, равноизмеримая с $|f|$. Перестановка $f^{*}$ может быть задана равенством

$$
f^{*}(t)=\sup _{|E|=t} \inf _{x \in E}|f(x)|, \quad 0<t<\infty
$$

Справедливо соотношение [13; гл. 2, § 2]

$$
\sup _{|E|=t} \int_{E}|f(x)| d x=\int_{0}^{t} f^{*}(u) d u
$$


что равносильно

$$
\inf _{|E|=t} \int_{c E}|f(x)| d x=\int_{t}^{\infty} f^{*}(u) d u
$$

Заметим, что

$$
f^{*}(2 t) \leqslant \frac{1}{t} \int_{t}^{\infty} f^{*}(u) d u, \quad t>0 .
$$

В дальнейшем обозначаем

$$
\begin{aligned}
f^{* *}(t) & =\frac{1}{t} \int_{0}^{t} f^{*}(u) d u \\
\bar{f}^{*}(t) & =\frac{1}{t} \int_{t}^{\infty} f^{*}(u) d u, \quad t>0 .
\end{aligned}
$$

В силу неравенства Харди [14; с. 124]

$$
\left\|f^{* *}\right\|_{p} \leqslant c_{p}\|f\|_{p}, \quad 1<p<\infty
$$

Основные свойства перестановок, которые мы будем использовать, изложены в [13], [14], [1]. Здесь отметим следующее свойство [13; гл. 2, § 2].

Лемма 1. Если последовательность $\left\{f_{k}\right\} \subset S_{0}$ сходится по мере $к$ функиии $f \in S_{0}$, то $f_{k}^{*}(t) \rightarrow f^{*}(t)$ в кажсдой точке непрерывности $f$.

Рассмотрим теперь перестановки по отдельным переменным и повторные перестановки. Пусть $x=\left(x_{1}, \ldots, x_{n}\right)$. Удаляя из набора $x$ координату $x_{k}$, мы получим $(n-1)$-мерный вектор, которьй будем обозначать через $\widehat{x}_{k}$. При этом будем писать $x=\left(x_{k}, \widehat{x}_{k}\right)$. Далее, через $\left(\tau, \widehat{x}_{k}\right)^{\prime}(\tau \in \mathbb{R})$ мы обозначаем вектор, у которого первая координата равна $\tau$, а затем следуют координаты $(n-1)$-мерного вектоpa $\widehat{x}_{k}$.

Предположим, что $f \in S_{0}\left(\mathbb{R}^{n}\right)$ и $1 \leqslant k \leqslant n$. Фиксируем $\widehat{x}_{k} \in \mathbb{R}^{n-1}$ и рассмотрим функцию переменного $x_{k}$

$$
f_{\widehat{x}_{k}}\left(x_{k}\right)=f\left(x_{k}, \widehat{x}_{k}\right), \quad x_{k} \in \mathbb{R} .
$$

Для почти всех $\widehat{x}_{k} \in \mathbb{R}^{n-1}$ имеем $f_{\widehat{x}_{k}} \in S_{0}(\mathbb{R})$. Обозначим

$$
\mathscr{R}_{k} f\left(\left(\tau, \widehat{x}_{k}\right)^{\prime}\right)=f_{\widehat{x}_{k}}^{*}(\tau), \quad \tau \in \mathbb{R}_{+}
$$

$\Phi$ ункция $\mathscr{R}_{k} f$ определена почти всюду на $\mathbb{R}_{+} \times \mathbb{R}^{n-1} ;$ будем называть ее $n е р е-$ становкой функции $f$ по $k$-й переменной. Используя приближение ступенчатыми функциями, лемму 1 и теорему Фубини, нетрудно убедиться в том, что $\mathscr{R}_{k} f$ является измеримой функцией, равноизмеримой с $|f|$ (см. также [15], [16]). Для любого набора $\left\{k_{1}, \ldots, k_{\nu}\right\}$ попарно различных индексов $1 \leqslant k_{j} \leqslant n$ полагаем $\mathscr{R}_{k_{1}, \ldots, k_{\nu}} f=\mathscr{R}_{k_{\nu}} \ldots \mathscr{R}_{k_{1}} f$. Далее, обозначим через $\mathscr{P}_{n}$ совокупность всех перестановок $\sigma=\left\{k_{1}, \ldots, k_{n}\right\}$ множества $\{1, \ldots, n\}$. Для любой $\sigma \in \mathscr{P}_{n}$ будем называть функцию

$$
\mathscr{R}_{\sigma} f(t) \equiv \mathscr{R}_{k_{1}, \ldots, k_{n}} f(t), \quad t \in \mathbb{R}_{+}^{n}
$$

$\mathscr{R}_{\sigma}$-перестановкой функции $f$. Таким образом, мы получаем $\mathscr{R}_{\sigma} f$, последовательно "переставляя" функцию $f$ в невозрастающем порядке по переменным $x_{k_{1}}, \ldots, x_{k_{n}}$. 
Легко видеть, что $\mathscr{R}_{\sigma} f$ монотонно убывает по каждой из переменных. В силу сделанного выше замечания $\mathscr{R}_{\sigma} f$ равноизмерима с $|f|$.

Далее, нетрудно убедиться в справедливости неравенств

$$
\begin{gathered}
\mathscr{R}_{\sigma} f(t) \leqslant f^{*}\left(t_{1} \cdots t_{n}\right), \\
\mathscr{R}_{\sigma}(f+g)(t+s) \leqslant \mathscr{R}_{\sigma} f(t)+\mathscr{R}_{\sigma} g(s) \quad\left(t, s \in \mathbb{R}_{+}^{n}\right) .
\end{gathered}
$$

Используя эти неравенства, мы точно так же, как в случае обычных перестановок $[13 ;$ гл. $2, \S 2]$, получаем следующий аналог леммы 1.

Лемма 2. Пусть $f_{k} \in S_{0}\left(\mathbb{R}^{n}\right)(k=1,2, \ldots)$ и последовательность $\left\{f_{k}\right\}$ сходится по мере на $\mathbb{R}^{n} \kappa$ функиии $f \in S_{0}\left(\mathbb{R}^{n}\right)$. Тогда для любой перестановки $\sigma \in \mathscr{P}_{n}$

$$
\lim _{k \rightarrow \infty} \mathscr{R}_{\sigma} f_{k}(t)=\mathscr{R}_{\sigma} f(t) \quad \text { для } \quad \text { n.в. } \quad t \in \mathbb{R}_{+}^{n} .
$$

В дальнейшем мы будем использовать некоторые средние, определяемые в терминах повторных перестановок.

Пусть $f \in S_{0}\left(\mathbb{R}^{n}\right)$. Обозначим (см. (2.4))

$$
\mathscr{R}_{k}^{*} f\left(\left(\tau, \widehat{x}_{k}\right)^{\prime}\right)=f_{\widehat{x}_{k}}^{* *}(\tau) \quad\left(\tau \in \mathbb{R}_{+}, \widehat{x}_{k} \in \mathbb{R}^{n-1}\right) .
$$

Далее, для любого $\sigma=\left\{k_{1}, \ldots, k_{n}\right\} \in \mathscr{P}_{n}$ полагаем

$$
\mathscr{R}_{\sigma}^{*} f(t)=\mathscr{R}_{k_{n}}^{*} \cdots \mathscr{R}_{k_{1}}^{*} f(t), \quad t \in \mathbb{R}_{+}^{n} .
$$

В силу (2.6)

$$
\left\|\mathscr{R}_{\sigma}^{*} f\right\|_{p} \leqslant c_{p}\|f\|_{p}, \quad 1 \leqslant p \leqslant \infty .
$$

Определим также следуюшие средние (см. (2.5)):

$$
\overline{\mathscr{R}}_{k} f\left(\left(\tau, \widehat{x}_{k}\right)^{\prime}\right)=\bar{f}_{\widehat{x}_{k}}^{*}(\tau) \quad\left(\tau \in \mathbb{R}_{+}, \widehat{x}_{k} \in \mathbb{R}^{n-1}\right)
$$

и

В силу (2.3)

$$
\overline{\mathscr{R}}_{\sigma} f(t)=\overline{\mathscr{R}}_{k_{n}} \ldots \overline{\mathscr{R}}_{k_{1}} f(t), \quad t \in \mathbb{R}_{+}^{n} .
$$

$$
\mathscr{R}_{\sigma} f(2 t) \leqslant \overline{\mathscr{R}}_{\sigma} f(t), \quad t \in \mathbb{R}_{+}^{n} .
$$

Рассмотрим некоторые оценки перестановок. Прежде всего, мы будем использовать следующую лемму (см. [1], [6]).

Лемма 3. Пусть $f \in L_{\mathrm{loc}}(\mathbb{R}), k \in \mathbb{N} u 0<\tau<\infty$. Тогда для любого $x \in \mathbb{R}$

$$
|f(x)| \leqslant\left(2^{k}-1\right) f^{*}(\tau)+\frac{1}{\tau} \int_{0}^{(k+1) \tau}\left|\Delta^{k}(h) f(x)\right| d h .
$$

Если, кроме того, функция $f$ имеет локально абсолютно непрерывную производную $f^{(k-1)}$, то

$$
|f(x)| \leqslant\left(2^{k}-1\right) f^{*}(\tau)+[(k+1) \tau]^{k-1} \int_{x}^{x+k(k+1) \tau}\left|f^{(k)}(\xi)\right| d \xi .
$$

Для любого $\sigma \in \mathscr{P}_{n}$ и $1 \leqslant \nu<\infty$ положим

$$
\overline{\mathscr{R}}_{\sigma}^{(\nu)} f(t)=\left(\overline{\mathscr{R}}_{\sigma} f^{\nu}(t)\right)^{1 / \nu} .
$$

Если $1 \leqslant p<\nu$ и $f \in L^{p}\left(\mathbb{R}^{n}\right)$, то

$$
\left\|\overline{\mathscr{R}}_{\sigma}^{\nu} f\right\|_{p} \leqslant c\|f\|_{p} .
$$

Для доказательства достаточно применить неравенство типа Харди (см., например, [17; неравенство (2.14)]). 
Лемма 4. Пусть $1 \leqslant p<\infty, 1 \leqslant \nu<p^{\prime}, 1 \leqslant k \leqslant n(n \geqslant 2) u \sigma \in \mathscr{P}_{n}$, $\sigma=\left\{j_{1}, \ldots, j_{n}\right\}$. Предположим, что $\varphi \in L^{\nu}(\mathbb{R})$ u $g \in L^{p}\left(\mathbb{R}^{n}\right)$. Пусть $k=j_{m}$

$u$

$$
f(x)=\int_{\mathbb{R}} \varphi\left(x_{k}-y\right) g\left(y, \widehat{x}_{k}\right) d y \text {. }
$$

Tогда:

(1) если $p>1 u \nu=1$, mo

$$
\mathscr{R}_{\sigma} f(t) \leqslant\|\varphi\|_{1} \mathscr{R}_{\sigma}^{*} g(t)
$$

(2) если $p=1 u \nu \geqslant 1$, mo

$$
\mathscr{R}_{\sigma} f(t) \leqslant\|\varphi\|_{\nu} \overline{\mathscr{R}}_{\widehat{\sigma}_{m}}^{(\nu)} \psi_{k}\left(\frac{\widehat{t}_{m}}{2}\right) t_{m}^{-1 / \nu}
$$

əде

$$
\psi_{k}\left(\widehat{x}_{k}\right)=\int_{\mathbb{R}}|g(x)| d x_{k}
$$

ДоказАТЕЛЬство. Сушествование свертки (2.15) для почти всех $x \in \mathbb{R}^{n}$ следует из теоремы Юнга.

Пусть для определенности $\sigma=\{1, \ldots, n\}$ и $1<k \leqslant n$. Предположим также, что функции $g$ и $\varphi$ неотрицательны.

Для любого множества $E \subset \mathbb{R}$ с $|E|=t_{1}$ имеем

$$
\begin{aligned}
\int_{E} f(x) d x_{1} & =\int_{\mathbb{R}} \varphi(y) d y \int_{E} g\left(x_{k}-y, \widehat{x}_{k}\right) d x_{1} \\
& \leqslant \int_{\mathbb{R}} \varphi(y) d y \int_{0}^{t_{1}} \mathscr{R}_{1} g\left(u_{1}, x_{2}, \ldots, x_{k}-y, \ldots, x_{n}\right) d u_{1} .
\end{aligned}
$$

Следовательно (см. (2.1)),

$$
\mathscr{R}_{1} f\left(t_{1}, x_{2}, \ldots, x_{n}\right) \leqslant \int_{\mathbb{R}} \varphi(y) \mathscr{R}_{1}^{*} g\left(t_{1}, x_{2}, \ldots, x_{k}-y, \ldots, x_{n}\right) d y
$$

Аналогично,

$$
\begin{aligned}
& \mathscr{R}_{1, \ldots, k-1} f\left(t_{1}, \ldots, t_{k-1}, x_{k}, \ldots, x_{n}\right) \\
& \quad \leqslant \int_{\mathbb{R}} \varphi(y) \mathscr{R}_{k-1}^{*} \ldots \mathscr{R}_{1}^{*} g\left(t_{1}, \ldots, t_{k-1}, x_{k}-y, \ldots, x_{n}\right) d y .
\end{aligned}
$$

Интегрируя по переменной $x_{k}$ по произвольному множеству $E \subset \mathbb{R}$ c $|E|=t_{k}$, получаем

$$
\begin{aligned}
& \mathscr{R}_{1, \ldots, k} f\left(t_{1}, \ldots, t_{k}, x_{k+1}, \ldots, x_{n}\right) \\
& \quad \leqslant\|\varphi\|_{1} \mathscr{R}_{k}^{*} \ldots \mathscr{R}_{1}^{*} g\left(t_{1}, \ldots, t_{k}, x_{k+1}, \ldots, x_{n}\right) .
\end{aligned}
$$

Отсюда следует (2.16). 
Пусть теперь $p=1, \nu \geqslant 1$. Последовательно применяя (2.3) и (2.2) к перестановкам по переменньм $x_{1}, \ldots, x_{k-1}$, получаем

$$
\begin{aligned}
& {\left[\mathscr{R}_{1, \ldots, k-1} f\left(2 t_{1}, \ldots, 2 t_{k-1}, x_{k}, \ldots, x_{n}\right)\right]^{\nu}} \\
& \quad \leqslant \frac{1}{t_{1} \cdots t_{k-1}} \inf _{\left|E_{k-1}\right|=t_{k-1}} \int_{c E_{k-1}} d x_{k-1} \cdots \inf _{\left|E_{1}\right|=t_{1}} \int_{c E_{1}} f^{\nu}(x) d x_{1} .
\end{aligned}
$$

Далее,

$$
\begin{aligned}
& {\left[\mathscr{R}_{1, \ldots, k} f\left(2 t_{1}, \ldots, 2 t_{k}, x_{k+1}, \ldots, x_{n}\right)\right]^{\nu}} \\
& \quad \leqslant \frac{1}{2 t_{k}} \sup _{|A|=2 t_{k}} \int_{A}\left[\mathscr{R}_{1, \ldots, k-1} f\left(2 t_{1}, \ldots, 2 t_{k-1}, x_{k}, \ldots, x_{n}\right)\right]^{\nu} d x_{k} \\
& \quad \leqslant \frac{1}{2 t_{1} \cdots t_{k}} \sup _{|A|=2 t_{k}\left|E_{k-1}\right|=t_{k-1}} \int_{c E_{k-1}} d x_{k-1} \cdots \inf _{\left|E_{1}\right|=t_{1}} \int_{c E_{1}} d x_{1} \int_{A} f^{\nu}(x) d x_{k} .
\end{aligned}
$$

В силу неравенства Минковского

$$
\left(\int_{A} f^{\nu}(x) d x_{k}\right)^{1 / \nu} \leqslant\|\varphi\|_{\nu} \psi_{k}\left(\widehat{x}_{k}\right)
$$

Следовательно,

$$
\begin{aligned}
& {\left[\mathscr{R}_{1, \ldots, k} f\left(2 t_{1}, \ldots, 2 t_{k}, x_{k+1}, \ldots, x_{n}\right)\right]^{\nu}} \\
& \quad \leqslant \frac{1}{2 t_{k}}\|\varphi\|_{\nu}^{\nu} \overline{\mathscr{R}}_{k-1} \ldots \overline{\mathscr{R}}_{1} \psi_{k}^{\nu}\left(t_{1}, \ldots, t_{k-1}, x_{k+1}, \ldots, x_{n}\right) .
\end{aligned}
$$

Отсюда следует (2.17). Лемма доказана.

Пусть $0<q, p<\infty$. Функция $f \in S_{0}\left(\mathbb{R}^{n}\right)$ принадлежит пространству Лоренца $L^{q, p}\left(\mathbb{R}^{n}\right)$, если

$$
\|f\|_{q, p} \equiv\left(\int_{0}^{\infty}\left(t^{1 / q} f^{*}(t)\right)^{p} \frac{d t}{t}\right)^{1 / p}<\infty
$$

Справедливо неравенство [14; с. 217]

$$
\|f\|_{q, s} \leqslant c\|f\|_{q, p} \quad(0<p<s<\infty)
$$

так что $L^{q, p} \subset L^{q, s}$ при $p<s$. В частности, при $0<p \leqslant q$

$$
L^{q, p} \subset L^{q, q} \equiv L^{q} .
$$

В дальнейшем полагаем

$$
\pi(t)=\prod_{k=1}^{n} t_{k}, \quad t \in \mathbb{R}_{+}^{n} .
$$

Пусть $0<q, p<\infty$ и $\sigma \in \mathscr{P}_{n}(n \geqslant 2)$. Через $L_{\mathscr{R}_{\sigma}}^{q, p}\left(\mathbb{R}^{n}\right)$ обозначим класс всех функций $f \in S_{0}\left(\mathbb{R}^{n}\right)$, для которых

$$
\|f\|_{q, p ; \mathscr{R}_{\sigma}} \equiv\left(\int_{\mathbb{R}_{+}^{n}}\left[\pi(t)^{1 / q} \mathscr{R}_{\sigma} f(t)\right]^{p} \frac{d t}{\pi(t)}\right)^{1 / p}<\infty
$$


(см. [15]). Полагаем также

$$
L_{\mathscr{R}}^{q, p}\left(\mathbb{R}^{n}\right)=\bigcap_{\sigma \in \mathscr{P}_{n}} L_{\mathscr{R}_{\sigma}}^{q, p}\left(\mathbb{R}^{n}\right), \quad\|f\|_{q, p ; \mathscr{R}}=\sum_{\sigma \in \mathscr{P}_{n}}\|f\|_{q, p ; \mathscr{R}_{\sigma}}
$$

Если $q>p$, то для любого $\sigma \in \mathscr{P}_{n}$ и любой функции $f \in S_{0}\left(\mathbb{R}^{n}\right)$

$$
\|f\|_{q, p} \leqslant c\|f\|_{q, p ; \mathscr{R}_{\sigma}}
$$

(см. [16]). Таким образом,

$$
L_{\mathscr{R}_{\sigma}}^{q, p} \subset L^{q, p} \quad(q>p) .
$$

Более того, это вложение является строгим [16].

\section{§ 3. Вложения в пространства $L_{\mathscr{R}}^{q, p}$}

ТЕОРема 1. Пусть $r_{1}, \ldots, r_{n}-$ положсительные числа $(n \geqslant 2)$,

$$
r \equiv n\left(\sum_{k=1}^{n} \frac{1}{r_{k}}\right)^{-1}<n, \quad 1 \leqslant p<\frac{n}{r} \quad u \quad q^{*}=\frac{n p}{n-r p} .
$$

Тогда для кажсдой функиии $f \in L_{p}^{r_{1}, \ldots, r_{n}}\left(\mathbb{R}^{n}\right)$

$$
\|f\|_{q^{*}, p ; \mathscr{R}} \leqslant c \sum_{j=1}^{n}\left\|D_{j}^{r_{j}} f\right\|_{p}
$$

ДокаЗАТЕльство. Пусть $f \in L_{p}^{r_{1}, \ldots, r_{n}}$. Обозначим через $\bar{r}_{k}$ наименьшее целое такое, что $r_{k} \leqslant \bar{r}_{k}(k=1, \ldots, n)$. Фиксируем $t \in \mathbb{R}_{+}^{n}$. В силу леммы 3 для любых $K>1,1 \leqslant j \leqslant n$ и $x \in \mathbb{R}^{n}$



где

$$
\Phi_{j}(x)=\frac{1}{t_{j}} \int_{0}^{t_{j}^{\prime}}\left|\Delta_{j}^{\bar{r}_{j}}(h) f(x)\right| d h, \quad t_{j}^{\prime}=\left(\bar{r}_{j}+1\right) K t_{j}
$$

(напомним, что через $\left(x_{j}, \widehat{x}_{j}\right)^{\prime}$ мы обозначаем вектор, полученный из вектора $x$ переносом $j$-й координаты на первое место). Пусть $\sigma \in \mathscr{P}_{n}$; для простоты будем считать, что $\sigma=\{1, \ldots, n\}$. В силу $(3.2)$

$$
\mathscr{R}_{\sigma} f(t) \leqslant 2^{\bar{r}_{j}} \mathscr{R}_{\sigma_{j}^{\prime}} f\left(\left(K t_{j}, \frac{\widehat{t}_{j}}{2}\right)^{\prime}\right)+\mathscr{R}_{\sigma} \Phi_{j}\left(\frac{t}{2}\right),
$$

где $\sigma_{j}^{\prime}$ получается из $\sigma$ перестановкой $j$-го индекса на первое место.

Обозначим $g_{j}=\left|D_{j}^{r_{j}} f\right|$. Если $r_{j}$ целое, то

$$
\begin{aligned}
\left|\Delta_{j}^{r_{j}}(h) f(x)\right| & \leqslant \int_{0}^{h} \cdots \int_{0}^{h} g_{j}\left(x+\left(u_{1}+\cdots+u_{r_{j}}\right) e_{j}\right) d u_{1} \cdots d u_{r_{j}} \\
& \leqslant h^{r_{j}-1} \int_{0}^{r_{j} h} g_{j}\left(x+y e_{j}\right) d y
\end{aligned}
$$


таким образом,

$$
\Phi_{j}(x) \leqslant c(K) t_{j}^{r_{j}-1} \int_{0}^{r_{j} t_{j}^{\prime}} g_{j}\left(x_{j}+y, \widehat{x}_{j}\right) d y .
$$

В случае дробного $r_{j}$ имеем

$$
\left|\Delta_{j}^{\bar{r}_{j}}(h) f(x)\right| \leqslant \int_{\mathbb{R}} g_{j}\left(x_{j}-y, \widehat{x}_{j}\right)\left|\Delta_{j}^{\bar{r}_{j}}(h) G_{r_{j}}(y)\right| d y
$$

$\left(G_{r_{j}}-\right.$ ядро Бесселя). Следовательно,

$$
\Phi_{j}(x) \leqslant \int_{\mathbb{R}} \varphi_{j}(y) g_{j}\left(x_{j}-y, \widehat{x}_{j}\right) d y
$$

где

$$
\varphi_{j}(y)=\frac{1}{t_{j}} \int_{0}^{t_{j}^{\prime}}\left|\Delta_{j}^{\bar{r}_{j}}(h) G_{r_{j}}(y)\right| d h .
$$

Используя оценки производных ядер $G_{\alpha}$, мы легко получаем (как в $[1 ;$ с. 160$]$ ), что для $1 \leqslant \nu<1 /\left(\bar{r}_{j}-r_{j}\right)$

$$
\left\|\Delta_{j}^{\bar{r}_{j}}(h) G_{r_{j}}\right\|_{\nu} \leqslant c h^{r_{j}+1 / \nu-1}, \quad h>0 .
$$

Предположим сначала, что $p>1$. В силу неравенств $(3.4),(3.5),(2.16)$ и (3.6) имеем для любого $1 \leqslant j \leqslant n$

$$
\mathscr{R}_{\sigma} \Phi_{j}(t) \leqslant c(K) t_{j}^{r_{j}} \mathscr{R}_{\sigma}^{*} g_{j}(t)
$$

Пусть $S=\|f\|_{q^{*}, p ; \mathscr{R}}$. Учитывая, что $C_{0}^{\infty}$ всюду плотно в $L_{p}^{r_{1}}, \ldots, r_{n}$, и используя лемму 2 , можем предполагать, что $S<\infty$. Далее, обозначим:

$$
A_{j}=\left\{t \in \mathbb{R}_{+}^{n}: t_{j} \leqslant \pi(t)^{r /\left(n r_{j}\right)}\right\}
$$

Тогда

$$
\bigcup_{j=1}^{n} A_{j}=\mathbb{R}_{+}^{n}
$$

Пользуясь неравенствами (3.3) и (3.7), имеем:

$$
\begin{aligned}
Q_{j}(\sigma) & \equiv\left(\int_{A_{j}} \pi(t)^{p / q^{*}-1} \mathscr{R}_{\sigma} f(t)^{p} d t\right)^{1 / p} \\
& \leqslant 2^{\bar{r}_{j}+n} K^{-1 / q^{*}} S+c(K)\left(\int_{A_{j}} \pi(t)^{p / q^{*}-1} t_{j}^{p r_{j}} \mathscr{R}_{\sigma}^{*} g_{j}(t)^{p} d t\right)^{1 / p} \\
& \leqslant 2^{\bar{r}_{j}+n} K^{-1 / q^{*}} S+c(K)\left(\int_{\mathbb{R}_{+}^{n}} \mathscr{R}_{\sigma}^{*} g_{j}(t)^{p} d t\right)^{1 / p} .
\end{aligned}
$$

В силу (2.8)

$$
\left(\int_{\mathbb{R}_{+}^{n}} \mathscr{R}_{\sigma}^{*} g_{j}(t)^{p} d t\right)^{1 / p} \leqslant c_{p}\left\|g_{j}\right\|_{p} \quad(p>1)
$$


Поэтому

$$
\|f\|_{q, p ; \mathscr{R}_{\sigma}} \leqslant \sum_{j=1}^{n} Q_{j}(\sigma) \leqslant c_{0} K^{-1 / q^{*}} S+c(K) \sum_{j=1}^{n}\left\|g_{j}\right\|_{p},
$$

где $c_{0}=\sum_{j=1}^{n} 2^{\bar{r}_{j}+n}$. Аналогичные неравенства справедливы для любого $\sigma \in \mathscr{P}_{n}$. Следовательно,

$$
S=\sum_{\sigma \in \mathscr{P}_{n}}\|f\|_{q, p ; \mathscr{R}_{\sigma}} \leqslant n ! c_{0} K^{-1 / q^{*}} S+c^{\prime}(K) \sum_{j=1}^{n}\left\|D_{j}^{r_{j}} f\right\|_{p} .
$$

Полагая $K=\left(2 n ! c_{0}\right)^{q^{*}}$, получаем $(3.1)$.

Пусть теперь $p=1$. Выберем $1<\nu<\min _{j}\left(\bar{r}_{j}-r_{j}\right)^{-1}$. В силу (3.4), (3.5), $(2.17)$ и (3.6) имеем $(\sigma=\{1, \ldots, n\})$

$$
\mathscr{R}_{\sigma} \Phi_{j}(t) \leqslant c(K) t_{j}^{r_{j}-1} F_{j}\left(\widehat{t}_{j}\right) \quad(j=1, \ldots, n)
$$

где

$$
F_{j}\left(\widehat{t}_{j}\right)=\overline{\mathscr{R}}_{\widehat{\sigma}_{j}}^{(\nu)} \psi_{j}\left(\widehat{t}_{j}\right), \quad \psi_{j}\left(\widehat{x}_{j}\right)=\int_{\mathbb{R}} g_{j}(x) d x_{j}
$$

Пользуясь неравенствами (3.3) и (3.10), получаем

$$
\begin{aligned}
Q_{j}(\sigma) & \equiv \int_{A_{j}} \pi(t)^{1 / q^{*}-1} \mathscr{R}_{\sigma} f(t) d t \\
& \leqslant 2^{\bar{r}_{j}+n} K^{-1 / q^{*}} S+c(K) \int_{A_{j}} \pi(t)^{1 / q^{*}-1} t_{j}^{r_{j}-1} F_{j}\left(\frac{\widehat{t}_{j}}{2}\right) d t .
\end{aligned}
$$

Последний интеграл равен $\left(\gamma_{j} \equiv r /\left(n r_{j}-r\right)>0\right)$

$$
\begin{array}{r}
\int_{\mathbb{R}_{+}^{n-1}} \pi\left(\widehat{t}_{j}\right)^{1 / q^{*}-1} F_{j}\left(\frac{\widehat{t}_{j}}{2}\right) d \widehat{t}_{j} \int_{0}^{\pi\left(\widehat{t}_{j}\right)^{\gamma_{j}}} t_{j}^{1 / q^{*}+r_{j}-2} d t_{j} \\
=c \int_{\mathbb{R}_{+}^{n-1}} F_{j}\left(\widehat{t}_{j}\right) d \widehat{t}_{j} \leqslant c^{\prime}\left\|\psi_{j}\right\|_{L\left(\mathbb{R}^{n-1}\right)}=c^{\prime}\left\|D_{j}^{r_{j}} f\right\|_{1}
\end{array}
$$

(заметим, что $1 / q^{*}+r_{j}>1$; мы использовали также неравенство (2.14)). Таким образом, мы вновь получаем (3.9) (c $p=1)$, откуда следует (3.1). Теорема доказана.

В силу (2.20) в условиях теоремы 1 справедливо неравенство

$$
\|f\|_{q^{*}, p} \leqslant c \sum_{j=1}^{n}\left\|D_{j}^{r_{j}} f\right\|_{p}
$$

Другое доказательство неравенства (3.11) содержится в [1].

Заметим, что в случае, когда все $r_{j}$ целые, доказательство теоремы 1 может быть упрощено. Действительно, в силу (2.12) имеем для любых $K>1,1 \leqslant j \leqslant n$, $1 \leqslant p<\infty$ и $x \in \mathbb{R}^{n}$




где

$$
\psi_{j}\left(\widehat{x}_{j}\right)=\left(\int_{\mathbb{R}}\left|D_{j}^{r_{j}} f(x)\right|^{p} d x_{j}\right)^{1 / p}
$$

Следовательно $(\sigma=\{1, \ldots, n\})$,

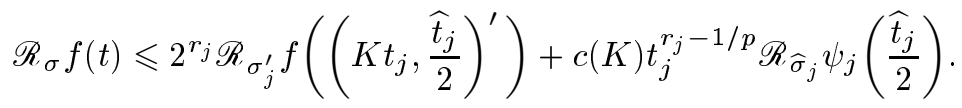

Отсюда, как и вьше,

$$
Q_{j}(\sigma) \leqslant 2^{r_{j}+n} K^{-1 / q^{*}} S+c(K)\left(\int_{A_{j}} \pi(t)^{p / q^{*}-1} t_{j}^{p r_{j}-1} \mathscr{R}_{\widehat{\sigma}_{j}} \psi_{j}\left(\frac{\widehat{t}_{j}}{2}\right)^{p} d t\right)^{1 / p}
$$

Применяя к последнему интегралу теорему Фубини, получаем, что этот интеграл равен

$$
c \int_{\mathbb{R}_{+}^{n-1}} \mathscr{R}_{\widehat{\sigma}_{j}} \psi_{j}\left(\widehat{t}_{j}\right)^{p} d \widehat{t}_{j}=c \int_{\mathbb{R}^{n}}\left|D_{j}^{r_{j}} f(x)\right|^{p} d x
$$

Таким образом, мы вновь придем к неравенству (3.9), откуда следует (3.1).

\section{§4. Оценки разностных норм}

Если $r>0$, то через $\bar{r}$ обозначаем наименьшее натуральное число такое, что $r \leqslant \bar{r}$.

ТЕОРемА 2. Пусть $r_{1}, \ldots, r_{n}-$ положсительные числа,

$$
r=n\left(\sum_{j=1}^{n} \frac{1}{r_{j}}\right)^{-1}, \quad 1 \leqslant p<q<\infty, \quad \varkappa=1-\frac{n}{r}\left(\frac{1}{p}-\frac{1}{q}\right)>0
$$

$u$

$$
\alpha_{j}=\varkappa r_{j} \quad(j=1, \ldots, n) .
$$

Если $1<p<\infty u n \geqslant 1$, или $p=1$ u $n \geqslant 2$, то для каждой функиии $f \in L_{p}^{r_{1}, \ldots, r_{n}}\left(\mathbb{R}^{n}\right)$ выполняются неравенства

$$
\begin{gathered}
\|f\|_{q, p ; \mathscr{R}} \leqslant c\|f\|_{L_{p}^{r_{1}, \ldots, r_{n}},} \\
\sum_{j=1}^{n}\left(\int_{0}^{\infty}\left[h^{-\alpha_{j}}\left\|\Delta_{j}^{\bar{r}_{j}}(h) f\right\|_{q, p ; \mathscr{R}}\right]^{p} \frac{d h}{h}\right)^{1 / p} \leqslant c \sum_{j=1}^{n}\left\|D_{j}^{r_{j}} f\right\|_{p} .
\end{gathered}
$$


ДокАЗАтЕльство. Докажем (4.2); неравенство (4.1) может быть доказано аналогично (и даже значительно проше).

Можем считать, что левая часть (4.2) конечна. Оценим, например, ее первое слагаемое. Обозначим

$$
f_{h}(x)=\Delta_{1}^{\bar{r}_{1}}(h) f(x) .
$$

Как и вьше, полагаем $g_{j}(x)=\left|D_{j}^{r_{j}} f(x)\right|$. Фиксируем $\sigma \in \mathscr{P}_{n}$.

Пусть сначала $p>1$. Если $r_{1}$ целое, то

$$
\left|f_{h}(x)\right| \leqslant h^{r_{1}-1} \int_{0}^{r_{1} h} g_{1}\left(x_{1}+y, \widehat{x}_{1}\right) d y .
$$

Если же $r_{1}$ нецелое, то

$$
\left|f_{h}(x)\right| \leqslant \int_{\mathbb{R}} g_{1}\left(x_{1}-y, \widehat{x}_{1}\right)\left|\Delta^{\bar{r}_{1}}(h) G_{r_{1}}(y)\right| d y .
$$

В обоих случаях, пользуясь неравенствами (2.16) и (3.6), получаем

$$
\mathscr{R}_{\sigma} f_{h}(t) \leqslant \operatorname{ch}^{r_{1}} \mathscr{R}_{\sigma}^{*} g_{1}(t)
$$

С другой стороны, пусть $1 \leqslant j \leqslant n$ и $m_{j}=\sigma^{-1}(j)$ (в перестановке $\sigma$ число $j$ находится на месте с номером $m_{j}$ ). Как и в теореме 1 , для любого $K>1$ имеем

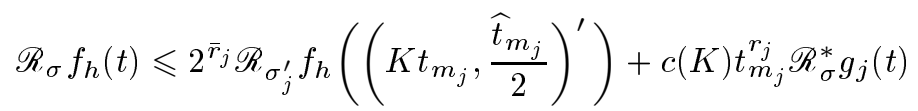

( $\sigma_{j}^{\prime}$ получается из $\sigma$ перестановкой числа $j$ на первое место).

Пусть

$$
J=\left(\int_{0}^{\infty}\left[h^{-\alpha_{1}}\left\|f_{h}\right\|_{q, p ; \mathscr{R}}\right]^{p} \frac{d h}{h}\right)^{1 / p} .
$$

Рассмотрим интеграл

$$
J(\sigma) \equiv\left(\int_{0}^{\infty} h^{-\alpha_{1} p-1} d h \int_{\mathbb{R}_{+}^{n}} \pi(t)^{p / q-1} \mathscr{R}_{\sigma} f_{h}(t)^{p} d t\right)^{1 / p} .
$$

Обозначим $s_{j}=n r_{j} / r$; тогда

$$
\sum_{j=1}^{n} \frac{1}{s_{j}}=1
$$

Прежде всего, в силу (4.5) и (2.8) имеем

$$
\begin{aligned}
J_{1}(\sigma)^{p} & \equiv \int_{0}^{\infty} h^{-\alpha_{1} p-1} d h \int_{\pi(t) \geqslant h^{s_{1}}} \pi(t)^{p / q-1} \mathscr{R}_{\sigma} f_{h}(t)^{p} d t \\
& \leqslant c \int_{0}^{\infty} h^{s_{1}(1-p / q)-1} d h \int_{\pi(t) \geqslant h^{s_{1}}} \pi(t)^{p / q-1} \mathscr{R}_{\sigma}^{*} g_{1}(t)^{p} d t \\
& =c \int_{\mathbb{R}_{+}^{n}} \pi(t)^{p / q-1} \mathscr{R}_{\sigma}^{*} g_{1}(t)^{p} d t \int_{0}^{\pi(t)^{1 / s_{1}}} h^{s_{1}(1-p / q)-1} d h \\
& =c^{\prime} \int_{\mathbb{R}_{+}^{n}} \mathscr{R}_{\sigma}^{*} g_{1}(t)^{p} d t \leqslant c^{\prime \prime}\left\|g_{1}\right\|_{p}^{p} .
\end{aligned}
$$


Далее, обозначим через $D(h, \sigma)$ множество таких $t \in \mathbb{R}_{+}^{n}$, для которых

$$
\mathscr{R}_{\sigma} f_{h}(t) \leqslant \sum_{j=1}^{n} 2^{\bar{r}_{j}+1} \mathscr{R}_{\sigma_{j}^{\prime}} f_{h}\left(\left(K t_{m_{j}}, \frac{\widehat{t}_{m_{j}}}{2}\right)^{\prime}\right)
$$

(постоянная $K$ будет выбрана ниже).

Пусть $t \notin D(h, \sigma)$. Тогда в силу (4.6) и (4.7)

$$
\mathscr{R}_{\sigma} f_{h}(t) \leqslant c(K) \pi(t)^{r / n} \prod_{j=1}^{n}\left(\mathscr{R}_{\sigma}^{*} g_{j}(t)\right)^{1 / s_{j}}
$$

Пользуясь этим неравенством и полагая

$$
E(h, \sigma)=\left\{t \in \mathbb{R}_{+}^{n}: \pi(t) \leqslant h^{s_{1}}, t \notin D(h, \sigma)\right\}
$$

имеем:

$$
\begin{aligned}
J_{2}(\sigma)^{p} & \equiv \int_{0}^{\infty} h^{-\alpha_{1} p-1} d h \int_{E(h, \sigma)} \pi(t)^{p / q-1} \mathscr{R}_{\sigma} f_{h}(t)^{p} d t \\
& \leqslant c(K) \int_{\mathbb{R}_{+}^{n}} \pi(t)^{p / q-1+p r / n} \prod_{j=1}^{n}\left(\mathscr{R}_{\sigma}^{*} g_{j}(t)\right)^{p / s_{j}} d t \int_{\pi(t)^{1 / s_{1}}}^{\infty} h^{-\alpha_{1} p-1} d h \\
& =c^{\prime}(K) \int_{\mathbb{R}_{+}^{n}} \prod_{j=1}^{n}\left(\mathscr{R}_{\sigma}^{*} g_{j}(t)\right)^{p / s_{j}} d t \\
& \leqslant c^{\prime}(K) \prod_{j=1}^{n}\left(\int_{\mathbb{R}_{+}^{n}} \mathscr{R}_{\sigma}^{*} g_{j}(t)^{p} d t\right)^{1 / s_{j}} \leqslant c^{\prime \prime}(K) \prod_{j=1}^{n}\left\|g_{j}\right\|_{p}^{p / s_{j}} .
\end{aligned}
$$

Наконец, в силу (4.8)

$$
J_{3}(\sigma) \equiv\left(\int_{0}^{\infty} h^{-\alpha_{1} p-1} d h \int_{D(h, \sigma)} \pi(t)^{p / q-1} \mathscr{R}_{\sigma} f_{h}(t)^{p} d t\right)^{1 / p} \leqslant c_{0} K^{-1 / q} J
$$

где $c_{0}=\sum_{j=1}^{n} 2^{\bar{r}_{j}+n}$.

Из полученных оценок следует, что для любого $\sigma \in \mathscr{P}_{n}$

$$
J(\sigma) \leqslant c_{0} K^{-1 / q} J+c(K) \sum_{j=1}^{n}\left\|g_{j}\right\|_{p}
$$

Суммируя по всем $\sigma \in \mathscr{P}_{n}$ и полагая $K=\left(2 c_{0} n !\right)^{q}$, получаем неравенство (4.2) для $p>1$.

Пусть теперь $p=1$. Выберем число $\nu>1$ так, чтобы выполнялись условия

$$
\nu<q, \quad \frac{1}{\nu}>\max _{j}\left(\bar{r}_{j}-r_{j}\right) .
$$


Снова фиксируем $\sigma \in \mathscr{P}_{n}$. В дальнейшем обозначаем $m_{j}=\sigma^{-1}(j), \eta_{j}=\widehat{\sigma}_{m_{j}}$ и

$$
\psi_{j}\left(\widehat{x}_{j}\right)=\int_{\mathbb{R}} g_{j}(x) d x_{j}, \quad F_{j}=\overline{\mathscr{R}}_{\eta_{j}}^{(\nu)} \psi_{j}
$$

В силу (2.14)

$$
\int_{\mathbb{R}_{+}^{n-1}} F_{j}(y) d y \leqslant c\left\|g_{j}\right\|_{1}
$$

Пользуясь неравенствами (4.3), (4.4), (2.17) и (3.6), имеем

$$
\mathscr{R}_{\sigma} f_{h}(t) \leqslant c h^{r_{1}-1 / \nu^{\prime}} t_{m_{1}}^{-1 / \nu} F_{1}\left(\widehat{t}_{m_{1}}\right)
$$

Кроме того, как и в теореме 1, для любых $1 \leqslant j \leqslant n$ и $K>1$



Пусть $\gamma(t)=\min \left(t_{m_{1}}, \pi\left(\widehat{t}_{m_{1}}\right)^{1 /\left(s_{1}-1\right)}\right)\left(t \in \mathbb{R}_{+}^{n}\right)$ и

$$
A(h)=\left\{t \in \mathbb{R}_{+}^{n}: \gamma(t) \geqslant h\right\}, \quad h>0 .
$$

Пользуясь неравенствами (4.11), (4.10) и условием $\nu<q$, имеем

$$
\begin{aligned}
\int_{0}^{\infty} & h^{-\alpha_{1}-1} d h \int_{A(h)} \pi(t)^{1 / q-1} \mathscr{R}_{\sigma} f_{h}(t) d t \\
\leqslant & c \int_{0}^{\infty} h^{s_{1}(1-1 / q)-1 / \nu^{\prime}-1} d h \int_{A(h)} \pi(t)^{1 / q-1} t_{m_{1}}^{-1 / \nu} F_{1}\left(\widehat{t}_{m_{1}}\right) d t \\
= & c \int_{\mathbb{R}_{+}^{n}} \pi(t)^{1 / q-1} t_{m_{1}}^{-1 / \nu} F_{1}\left(\widehat{t}_{m_{1}}\right) d t \int_{0}^{\gamma(t)} h^{s_{1}(1-1 / q)-1 / \nu^{\prime}-1} d h \\
= & c_{1} \int_{\mathbb{R}_{+}^{n}} \pi(t)^{1 / q-1} t_{m_{1}}^{-1 / \nu} F_{1}\left(\widehat{t}_{m_{1}}\right) \gamma(t)^{s_{1}(1-1 / q)-1 / \nu^{\prime}} d t \\
= & c_{1}\left(\int_{\mathbb{R}_{+}^{n-1}} \pi(y)^{1 / q-1} F_{1}(y) d y \int_{0}^{\pi(y)^{1 /\left(s_{1}-1\right)} \xi^{\left(s_{1}-1\right)(1-1 / q)-1} d \xi} \xi^{1 / q-1 / \nu-1} d \xi\right) \\
& +\int_{\mathbb{R}_{+}^{n-1}} \pi(y)^{(1 / \nu-1 / q) /\left(s_{1}-1\right)} F_{1}(y) d y \int_{\pi(y)^{1 /\left(s_{1}-1\right)}}^{\infty}{ }^{\infty} F_{1}(y) d y \leqslant c_{3}\left\|g_{1}\right\|_{1} . \\
= & c_{\mathbb{R}_{+}^{n-1}}
\end{aligned}
$$

Далее, положим $B(h)=\mathbb{R}_{+}^{n} \backslash A(h)$. Пусть $t \in B(h)$ и индекс $j=j(t)$ определяется из условия $t_{m_{j}}^{r_{j}}=\min _{k} t_{m_{k}}^{r_{k}}$. Тогда $t_{m_{j}}^{s_{j}-1} \leqslant \pi\left(\widehat{t}_{m_{j}}\right)$. Если $j=1$, то $t_{m_{1}} \leqslant h$ по определению $B(h)$. Пусть $j \geqslant 2$. Имеем $t_{m_{j}}^{r_{j} / r_{k}} \leqslant t_{m_{k}}(k=2, \ldots, n)$; отсюда

$$
t_{m_{j}}^{r_{j} / r_{1}} \leqslant \pi\left(\widehat{t}_{m_{1}}\right)^{1 /\left(s_{1}-1\right)} .
$$


Кроме того, $t_{m_{j}}^{r_{j} / r_{1}} \leqslant t_{m_{1}}$. Следовательно,

$$
t_{m_{j}}^{r_{j} / r_{1}} \leqslant \gamma(t) \leqslant h
$$

Таким образом, полагая

$$
B_{k}(h)=\left\{t \in \mathbb{R}_{+}^{n}: t_{m_{k}} \leqslant \min \left(h^{r_{1} / r_{k}}, \pi\left(\widehat{t}_{m_{k}}\right)^{1 /\left(s_{k}-1\right)}\right)\right\},
$$

имеем

$$
B(h) \subset \bigcup_{k=1}^{n} B_{k}(h) .
$$

Теперь, пользуясь оценкой (4.12), получаем для любого $k=1, \ldots, n$

$$
\begin{aligned}
& \int_{0}^{\infty} h^{-\alpha_{1}-1} d h \int_{B_{k}(h)} \pi(t)^{1 / q-1} \mathscr{R}_{\sigma} f_{h}(t) d t \\
& \quad \leqslant 2^{\bar{r}_{k}+n} K^{-1 / q} J+c(K) \int_{0}^{\infty} h^{-\alpha_{1}-1} d h \int_{B_{k}(h)} \pi(t)^{1 / q-1} t_{m_{k}}^{r_{k}-1} F_{k}\left(\frac{\widehat{t}_{m_{k}}}{2}\right) d t .
\end{aligned}
$$

Пусть $E_{k}(\xi)=\left\{y \in \mathbb{R}_{+}^{n-1}: \pi(y) \geqslant \xi^{s_{k}-1}\right\}, \xi \in \mathbb{R}_{+}$. Интеграл в правой части (4.13) равен

$$
\begin{aligned}
\int_{0}^{\infty} & h^{-\alpha_{1}-1} d h \int_{0}^{h^{r_{1} / r_{k}}} \xi^{r_{k}+1 / q-2} d \xi \int_{E_{k}(\xi)} \pi(y)^{1 / q-1} F_{k}\left(\frac{y}{2}\right) d y \\
= & c \int_{0}^{\infty} \xi^{\left(s_{k}-1\right)(1-1 / q)-1} d \xi \int_{E_{k}(\xi)} \pi(y)^{1 / q-1} F_{k}\left(\frac{y}{2}\right) d y \\
= & c^{\prime} \int_{\mathbb{R}_{+}^{n-1}} F_{k}(y) d y \leqslant c^{\prime \prime}\left\|g_{k}\right\|_{1} .
\end{aligned}
$$

Полученные оценки вновь дают неравенство (4.9) (с $p=1$ ), из которого, в свою очередь, следует (4.2). Теорема доказана.

Пользуясь неравенством (2.20), получаем, что в условиях теоремы 2

$$
\sum_{j=1}^{n}\left(\int_{0}^{\infty}\left[h^{-\alpha_{j}} \omega_{j}^{\bar{r}_{j}}(f ; h)_{q}\right]^{p} \frac{d h}{h}\right)^{1 / p} \leqslant c \sum_{j=1}^{n}\left\|D_{j}^{r_{j}} f\right\|_{p} .
$$

Иными словами, справедливо вложение

$$
L_{p}^{r_{1}, \ldots, r_{n}}\left(\mathbb{R}^{n}\right) \hookrightarrow B_{q, p}^{\alpha_{1}, \ldots, \alpha_{n}}\left(\mathbb{R}^{n}\right) .
$$

Здесь $1<p<\infty, n \geqslant 1$ или $p=1, n \geqslant 2$. Подчеркнем, что при $p=n=1$ вложение (4.15) не имеет места (см. [1], [17]).

Как уже отмечалось, в случае целых $r_{j}$ и $p>1$ вложение (4.15) доказал В.П. Ильин [5; теорема 18.12]; для целых $r_{j}$ и $p=1(n \geqslant 2)$ оно было доказано в работе [6] (см. также [17]).

Для пространств $L_{p}^{r_{1}, \ldots, r_{n}}$ при $p>1$ П.И. Лизоркиным [18] была установлена следуюшая теорема вложения разных метрик. 
ТЕОРЕМА. Пусть $r_{1}, \ldots, r_{n}-$ положительнье числа,

$$
r=n\left(\sum_{k=1}^{n} \frac{1}{r_{k}}\right)^{-1}, \quad 1<p<q<\infty \quad u \quad \varkappa \equiv 1-\frac{n}{r}\left(\frac{1}{p}-\frac{1}{q}\right)>0
$$

Tогда



При $p=n=1$ эта теорема теряет силу. Однако из (4.15) следует справедливость вложения (4.16) при $p=1, n \geqslant 2$. Действительно, для $q>1$ (см. [18], [19])

$$
B_{q, 1}^{\alpha_{1}, \ldots, \alpha_{n}}\left(\mathbb{R}^{n}\right) \hookrightarrow L_{q}^{\alpha_{1}, \ldots, \alpha_{n}}\left(\mathbb{R}^{n}\right) \quad\left(\alpha_{i}>0\right)
$$

Учитывая (4.15), получаем вложение (4.16) для $p=1, n \geqslant 2$.

\section{§5. Оценки преобразований Фурье}

Преобразование Фурье функции $f \in L^{1}\left(\mathbb{R}^{n}\right)$ определяется равенством

$$
\widehat{f}(\xi)=\int_{\mathbb{R}^{n}} f(x) e^{-2 \pi i x \xi} d x, \quad \xi \in \mathbb{R}^{n}
$$

Будем рассматривать оценки преобразований Фурье функций из пространств $L_{1}^{r_{1}, \ldots, r_{n}}$. Для случая целых $r_{k}$ аналогичные вопросы изучались в статье автора [7] (эта статья, в свою очередь, является продолжением работ Ж. Бургейна и А. Пелчинского, М. Войцеховского [12]). Основные результаты работы [7] устанавливаются с помощью неравенства (4.14), которое для целых $r_{k}$ было доказано в [6]. Эти результаты (теоремы 1 и 2 из [7]) без изменения переносятся на случай произвольных $r_{k}>0$. Однако мы докажем более сильное утверждение.

ТЕОРемА 3. Пусть $r_{1}, \ldots, r_{n}-$ положсительные числа $(n \geqslant 2)$,

$$
\lambda(\xi)=\sum_{j=1}^{n}\left(1+4 \pi^{2} \xi_{j}^{2}\right)^{r_{j} / 2}
$$

$r=n\left(\sum_{j=1}^{n} r_{j}^{-1}\right)^{-1}$ u $0<\varepsilon \leqslant \min (1, r / n)$. Тогда существует постоянная $c$, зависящая только от $\varepsilon$ и $r_{1}, \ldots, r_{n}$, такая, что для любой функиии $f \in$ $L_{1}^{r_{1}, \ldots, r_{n}}\left(\mathbb{R}^{n}\right)$ выполняется неравенство

$$
\sum_{\sigma \in \mathscr{P}_{n}} \int_{\mathbb{R}_{+}^{n}} \pi(t)^{\varepsilon-1} \mathscr{R}_{\sigma} \psi_{\varepsilon}(t) d t \leqslant c \sum_{k=1}^{n}\left\|D_{k}^{r_{k}} f\right\|_{1}
$$

əде $\psi_{\varepsilon}(\xi)=\widehat{f}(\xi) \lambda(\xi)^{1-\varepsilon n / r}$. 
ДокАЗАТЕльство. Прежде всего, заметим, что неравенство (5.1) тем сильнее, чем меньше $\varepsilon$. Действительно, пусть $s_{k}=n r_{k} / r$. В силу (4.7)

$$
\lambda(\xi) \geqslant \sum_{j=1}^{n}\left(2 \pi\left|\xi_{j}\right|\right)^{r} \geqslant(2 \pi)^{r} \min _{k} s_{k}\left(\prod_{j=1}^{n}\left|\xi_{j}\right|\right)^{r / n}
$$

Стало быть, для $0<\varepsilon_{1}<\varepsilon_{2}$

$$
\left|\psi_{\varepsilon_{2}}(\xi)\right| \leqslant c\left|\psi_{\varepsilon_{1}}(\xi)\right|\left(\prod_{j=1}^{n}\left|\xi_{j}\right|\right)^{\varepsilon_{1}-\varepsilon_{2}}
$$

$$
\mathscr{R}_{\sigma} \psi_{\varepsilon_{2}}(t) \leqslant c \mathscr{R}_{\sigma} \psi_{\varepsilon_{1}}\left(\frac{t}{2}\right) \pi(t)^{\varepsilon_{1}-\varepsilon_{2}},
$$

откуда и следует наше замечание.

Далее, не уменьшая общности, можем считать, что $f \in C_{0}^{\infty}\left(\mathbb{R}^{n}\right)$. Пусть $\rho_{\nu}=$ $r_{\nu}(1-\varepsilon n / r)$. Зададим функции $\varphi_{\nu}$ в терминах преобразований Фурье:

$$
\widehat{\varphi}_{\nu}(\xi)=\left(1+4 \pi^{2} \xi_{\nu}^{2}\right)^{\rho_{\nu} / 2} \widehat{f}(\xi) \quad(\nu=1, \ldots, n)
$$

Таким образом,

$$
f(x)=\int_{\mathbb{R}} G_{\rho_{\nu}}\left(x_{\nu}-y\right) \varphi_{\nu}\left(y, \widehat{x}_{\nu}\right) d y \quad(\nu=1, \ldots, n) .
$$

Пусть, например, $\sigma=\{1, \ldots, n\}$. Используя индукцию, нетрудно показать, что для любых $1 \leqslant k \leqslant n, t \in \mathbb{R}_{+}^{n}$ сушествует измеримое множество $E(t, k) \subset \mathbb{R}^{n}$, обладающее следующими свойствами:

$$
\begin{gathered}
|E(t, k)| \geqslant \frac{\pi(t)}{2}, \\
\left|\xi_{k}\right| \geqslant t_{k} / 4 \text { для любого } \xi \in E(t, k)
\end{gathered}
$$

и

$$
\left|\psi_{\varepsilon}(\xi)\right| \geqslant \mathscr{R}_{\sigma} \psi_{\varepsilon}(t) \text { для любого } \xi \in E(t, k) .
$$

Обозначим теперь

$$
A_{k}=\left\{t \in \mathbb{R}_{+}^{n}: t_{k}^{r_{k}}=\max _{1 \leqslant j \leqslant n} t_{j}^{r_{j}}\right\}
$$

Фиксируем $1 \leqslant k \leqslant n$ и $t \in A_{k}$. Положим $E \equiv E(t, k)$. Далее, пусть

$$
E^{(m)}=\left\{\xi \in E:\left|\xi_{m}\right|^{r_{m}}=\max _{1 \leqslant j \leqslant n}\left|\xi_{j}\right|^{r_{j}}\right\}, \quad m=1, \ldots, n
$$

В силу (5.4) сушествует такое $\nu \equiv \nu(t, k)$, что

$$
\left|E^{(\nu)}\right| \geqslant \frac{\pi(t)}{2 n}
$$


Учитывая (5.5), получаем, что

$$
\left|\xi_{\nu}\right| \geqslant\left|\xi_{k}\right|^{r_{k} / r_{\nu}} \geqslant\left(\frac{t_{k}}{4}\right)^{r_{k} / r_{\nu}} \text { для любого } \xi \in E^{(\nu)} .
$$

Наконец, в силу (5.6) и (5.8)

$$
\mathscr{R}_{\sigma} \psi_{\varepsilon}(t) \leqslant \frac{2 n}{\pi(t)} \int_{E^{(\nu)}}\left|\psi_{\varepsilon}(\xi)\right| d \xi \leqslant \frac{c}{\pi(t)} \int_{E^{(\nu)}}\left|\widehat{\varphi}_{\nu}(\xi)\right| d \xi
$$

Обозначим $\delta_{h}(x)=\varphi_{\nu}(x)-\varphi_{\nu}\left(x+h e_{\nu}\right)(h>0)$. Тогда

$$
\widehat{\delta}_{h}(\xi)=\widehat{\varphi}_{\nu}(\xi)\left(1-e^{2 \pi i h \xi_{\nu}}\right)
$$

Пусть $\tau=\left(4 / t_{k}\right)^{r_{k} / r_{\nu}}$. В силу (5.9) имеем для любого $\xi \in E^{(\nu)}$

$$
\frac{1}{\tau} \int_{0}^{\tau}\left|e^{2 \pi i h \xi_{\nu}}-1\right| d h \geqslant 1-\frac{1}{2 \pi}
$$

Поэтому, учитывая (5.10), имеем:

$$
\mathscr{R}_{\sigma} \psi_{\varepsilon}(t) \leqslant \frac{c}{\pi(t) \tau} \int_{0}^{\tau} d h \int_{E^{(\nu)}}\left|\widehat{\delta}_{h}(\xi)\right| d \xi .
$$

Можем считать, что $0<\varepsilon<1 / 2$. Тогда сушествует такое $1<p<2$, что

$$
0<\varepsilon-\frac{1}{p}<\min _{\nu} \frac{1}{s_{\nu}}
$$

В силу (5.8) и неравенства Хаусдорфа-Юнга

$$
\int_{E^{(\nu)}}\left|\widehat{\delta}_{h}(\xi)\right| d \xi \leqslant \pi(t)^{1 / p}\left\|\widehat{\delta}_{h}\right\|_{p^{\prime}} \leqslant \pi(t)^{1 / p}\left\|\delta_{h}\right\|_{p}
$$

Отсюда и из (5.11) следует, что

$$
\mathscr{R}_{\sigma} \psi_{\varepsilon}(t) \leqslant c \pi(t)^{-1 / p^{\prime}} \sum_{j=1}^{n} \omega_{j}\left(\varphi_{j} ; t_{k}^{-r_{k} / r_{j}}\right)_{p}, \quad t \in A_{k} .
$$

Пользуясь этой оценкой, получаем (см. (5.7)):

$$
\begin{aligned}
I_{k}(\sigma) & \equiv \int_{A_{k}} \pi(t)^{\varepsilon-1} \mathscr{R}_{\sigma} \psi_{\varepsilon}(t) d t \\
& \leqslant c \sum_{j=1}^{n} \int_{0}^{\infty} t_{k}^{n r_{k}\left(\varepsilon-1 / p^{\prime}\right) / r-1} \omega_{j}\left(\varphi_{j} ; t_{k}^{-r_{k} / r_{j}}\right)_{p} d t_{k} \\
& =c^{\prime} \sum_{j=1}^{\infty} \int_{0}^{\infty} z^{-n r_{j}\left(\varepsilon-1 / p^{\prime}\right) / r} \omega_{j}\left(\varphi_{j} ; z\right)_{p} \frac{d z}{z} \\
& =c^{\prime} \sum_{j=1}^{\infty} \int_{0}^{\infty} z^{\rho_{j}-\alpha_{j}} \omega_{j}\left(\varphi_{j} ; z\right)_{p} \frac{d z}{z}
\end{aligned}
$$


где $\alpha_{j}=r_{j}\left(1-n /\left(r p^{\prime}\right)\right)$. В силу $(5.12)$

$$
0<\alpha_{j}-\rho_{j}<1, \quad j=1, \ldots, n .
$$

Учитывая (5.3) и применяя теорему об изоморфизме пространств Бесова [3; гл. 5 , теорема $\left.4^{\prime}\right]$, имеем

$$
\int_{0}^{\infty} z^{\rho_{j}-\alpha_{j}} \omega_{j}\left(\varphi_{j} ; z\right)_{p} \frac{d z}{z} \leqslant c \int_{0}^{\infty} z^{-\alpha_{j}} \omega_{j}^{\bar{r}_{j}}(f ; z)_{p} \frac{d z}{z}
$$

Пользуясь неравенством (4.14), получаем

$$
I_{k}(\sigma) \leqslant c \sum_{j=1}^{n}\left\|D_{j}^{r_{j}} f\right\|_{1}, \quad k=1, \ldots, n
$$

Отсюда следует (5.1). Теорема доказана.

ЗАмечание 1. Теорема об изоморфизме, на которую мы сослались в связи с неравенством (5.13), доказана в [3] (см. также [2; теорема 8.9.1]) для $n$-мерных изотропных бесселевых потенциалов. Однако те же рассуждения применимы и в нашем случае, когда бесселев потенциал берется только по одной переменной. Кроме того, используя теорему об изоморфизме для одномерного случая, нетрудно получить оценку модуля непрерьвности функции $\varphi_{\nu}$ через модуль непрерывности (порядка $\bar{r}_{\nu}$ ) функции $f$, из которой, в свою очередь, следует неравенство (5.13).

Рассмотрим некоторые следствия теоремы 3 . Прежде всего, в силу (2.20) из (5.1) следует неравенство

$$
\int_{0}^{\infty} t^{\varepsilon-1} \psi_{\varepsilon}^{*}(t) d t \leqslant c \sum_{k=1}^{n}\left\|D_{k}^{r_{k}} f\right\|_{1}
$$

В частности, если $r \equiv n\left(\sum_{k=1}^{n} r_{k}^{-1}\right)^{-1} \leqslant n$, то, полагая $\varepsilon=r / n$, получаем неравенство

$$
\|\widehat{f}\|_{n / r, 1} \leqslant c \sum_{k=1}^{n}\left\|D_{k}^{r_{k}} f\right\|_{1}
$$

Далее, в силу неравенства Харди-Литтлвуда [14; с. 43] и (5.2) из (5.1) следует, что в условиях теоремы 3

$$
\int_{\mathbb{R}^{n}}|\widehat{f}(\xi)|\left(\sum_{j=1}^{n}\left|\xi_{j}\right|^{r_{j}}\right)^{1-n / r} d \xi \leqslant c \sum_{k=1}^{n}\left\|D_{k}^{r_{k}} f\right\|_{1} .
$$

Для целых $r_{j}$ неравенства (5.14) и (5.15) были доказаны в [7].

Предположим теперь, что $r_{k} \geqslant 1(k=1, \ldots, n)$. Пусть $\varepsilon=1 / n$ и $\psi(\xi)=$ $\lambda(\xi)^{1-1 / r} \widehat{f}(\xi)$. Из неравенств (5.1) и (2.20) следует, что

$$
\|\psi\|_{n} \leqslant c\|\psi\|_{n, 1} \leqslant c^{\prime} \sum_{k=1}^{n}\left\|D_{k}^{r_{k}}\right\|_{1}
$$


Далее, справедливо неравенство

$$
\lambda(\xi)^{(1-1 / r) n} \geqslant \prod_{j=1}^{n}\left|\xi_{j}\right|^{r_{j}-1}
$$

Действительно, полагая $\alpha_{j}=1-1 / r_{j}$, имеем

$$
\alpha \equiv \sum_{j=1}^{n} \alpha_{j}=\left(1-\frac{1}{r}\right) n
$$

остается воспользоваться неравенством

$$
\left(\sum_{j=1}^{n} \eta_{j}\right)^{\alpha} \geqslant \prod_{j=1}^{n} \eta_{j}^{\alpha_{j}}, \quad \eta_{j}=\left|\xi_{j}\right|^{r_{j}}
$$

Таким образом, мы получаем

СлеДСТВИЕ. Пусть $r_{k} \geqslant 1(k=1, \ldots, n ; n \geqslant 2)$. Тогда для любой функции $f \in L_{1}^{r_{1}, \ldots, r_{n}}\left(\mathbb{R}^{n}\right)$

$$
\left(\int_{\mathbb{R}^{n}}|\widehat{f}(\xi)|^{n} \prod_{j=1}^{n}\left|\xi_{j}\right|^{r_{j}-1} d \xi\right)^{1 / n} \leqslant c \sum_{j=1}^{n}\left\|D_{j}^{r_{j}} f\right\|_{1}
$$

Для $n=2$ и целых $r_{1}, r_{2}$ неравенство (5.16) было доказано другими методами в работе [11]. Заметим, что можно доказать (5.16) и прямым путем (по схеме доказательства теоремы 2 из [7]).

В следуюшем замечании ограничимся случаем целых $r_{k}$.

ЗАмечаниЕ 2. Пусть $r_{1}, \ldots, r_{n} \in \mathbb{N}(n \geqslant 2)$. Сумму в правой части $(5.16)$ можно заменить средним геометрическим. А именно для любой функции $f \in$ $W_{1}^{r_{1}, \ldots, r_{n}}\left(\mathbb{R}^{n}\right)$

$$
\int_{\mathbb{R}^{n}}|\widehat{f}(\xi)|^{n} \prod_{j=1}^{n}\left|\xi_{j}\right|^{r_{j}-1} d \xi \leqslant c \prod_{j=1}^{n}\left\|D_{j}^{r_{j}} f\right\|_{1}
$$

В самом деле, пусть $A_{k}=\left\|D_{k}^{r_{k}} f\right\|_{1}$. Если $A_{k}=0$ для некоторого $k$, то $f$ эквивалентна нулю. Пусть $A_{k}>0(k=1, \ldots, n)$. Положим

$$
g\left(x_{1}, \ldots, x_{n}\right)=f\left(\varepsilon_{1} x_{1}, \ldots, \varepsilon_{n} x_{n}\right), \quad \varepsilon_{k}=A_{k}^{-1 / r_{k}} .
$$

Применяя к функции $g$ неравенство (5.16), получаем (5.17).

Отметим, что подобные преобразования "аддитивных" оценок в "мультипликативные" хорошо известны (см. [2; гл. 7]). 


\section{Список литературы}

1. Kolyada V. I. Rearrangement of functions and embedding of anisotropic spaces of Sobolev type // East J. Approx. 1998. V. 4. № 2. P. 111-199.

2. Никольский C. M. Приближение функций многих переменных и теоремы вложения. М.: Наука, 1977.

3. Стейн И. М. Сингулярные интегралы и диффференциальные свойства функций. М.: Мир, 1973.

4. Самко С.Г., Килбас А.А., Маричев О.И. Интегралы и производные дробного порядка и некоторые их приложения. Минск: Наука и техника, 1987.

5. Бесов О. В., Ильин В. П., Никольский С. М. Интегральные представления функций и теоремы вложения. М.: Наука, 1975.

6. Коляда В. И. О вложении пространств Соболева // Матем. заметки. 1993. Т. 54. № 3. C. $48-71$

7. Kolyada V. I. Estimates of Fourier transforms in Sobolev spaces // Studia Math. 1997. V. 125. № 1. P. $67-74$.

8. Ульянов П. Л. Вложение некоторых классов функций $H_{p}^{\omega} / /$ Изв. АН СССР. Сер. матем. 1968. Т. 3. №4. С. 649-686.

9. Ульянов П. Л. Теоремы вложения и соотношения между наилучшими приближениями (модулями непрерывности) в разных метриках // Матем. сб. 1970. Т. 81. № 1. С. 104-131.

10. Освальд П. Модули непрерьвности равноизмеримых функций и приближение функций алгебраическими полиномами в $L^{p} / /$ Дисс. ... канд. физ.-матем. наук. Одесса: Одесский гос. ун-т, 1978.

11. Pelczyński A., Senator K. On isomorphisms of anisotropic Sobolev spaces with "classical Banach spaces" and a Sobolev type embedding theorem // Studia Math. 1986. V. 84. P. 169-215.

12. Pelczyński A., Wojciechowski M. Molecular decompositions and embedding theorems for vector-valued Sobolev spaces with gradient norm // Studia Math. 1993. V. 107. P. 61-100.

13. Крейн С.Г., Петунин Ю.И., Семенов Е.М. Интерполяция линейных операторов. М.: Наука, 1978.

14. Bennett C., Sharpley R. Interpolation of operators. Boston: Academic Press, 1988. (Pure Appl. Math. V. 129.)

15. Blozinski A. P. Multivariate rearrangements and Banach function spaces with mixed norms // Trans. Amer. Math. Soc. 1981. V. 263. № 1. P. 149-167.

16. Яченко А. А. Итеративные перестановки функций и пространства Лоренца // Изв. вузов. Сер. матем. 1998. Т. 432. № 5. С. 73-77.

17. Коляда В. И. О соотношениях между модулями непрерывности в разных метриках // Труды МИАН. 1988. Т. 181. С. 117-136.

18. Лизоркин П.И. Неизотропные бесселевы потенциалы. Теоремы вложения для пространств $L_{p}^{\left(r_{1}, \ldots, r_{n}\right)}$ с дробными производньми // Докл. АН СССР. 1966. Т. 170. № 3 . C. $508-511$.

19. Лизоркин П. И. Обобщенное лиувиллевское дифференцирование и метод мультипликаторов в теории вложений // Труды МИАН. 1969. Т. 105. С. 89-167.

Одесский государственнпй университет им. И.И. Мечникова,

Институт математики, экономики и механики, Одесса, Украина

E-mail: kolyada@paco.net
Поступила в редакцию

02.11 .2000 